\title{
A novel method for the absolute end-to-end calibration of the Auger fluo- rescence telescopes
}

\author{
Hermann-Josef Mathes ${ }^{1, *}$ for the Pierre Auger Collaboration \\ ${ }^{1}$ Karlsruhe Institute for Technology, Institute for Nuclear Physics, Hermann-von-Helmholtz-Platz 1, 76344 Eggenstein- \\ Leopoldshafen, Germany
}

\begin{abstract}
The fluorescence detector technique is using the atmosphere as a calorimeter. Besides the precise monitoring of the parameters of the atmosphere a proper knowledge of the optical properties in the UV range of all optical components involved in the measurements of the fluorescence light is vital.

Until now, the end-to-end calibration was performed with a $4.5 \mathrm{~m}^{2}$ large, uniformly lit light source attached to the aperture of the telescopes. To improve the maintainability we propose an alternative setup where a small and lightweight light source of known optical properties re-samples the measurement of the big light source piece by piece. This will be achieved by moving the light source based on an integrating sphere in two dimensions in front of the aperture. A prototype setup has been installed and we are currently optimizing the parameters of the system and the procedures. The aim is to reduce the effort for the procedure without diminishing the quality of the measurement.

First measurements with this setup have already been performed and the measurements of the geometrical and optical properties of the light source are an ongoing activity. We present our calibration scheme and the first, preliminary results.
\end{abstract}

\section{Introduction}

The fluorescence detector (FD) of the Pierre Auger Observatory [1] consists of 27 fluorescence telescopes at 4 different sites [2], [3] overlooking an area of $3000 \mathrm{~km}^{2}$ equipped with over 1600 water Cherenkov detectors. The FD measures the fluorescence light of extensive air showers on clear, moonless nights. The amount of measured fluorescence light is an indicator for the energy of the primary particle. However the variable optical features of the atmosphere, mainly attenuation and scattering, as well as the properties of the FD telescope are crucial for a precise measurement.

For the FD of the Pierre Auger Observatory various systems are used to monitor the variability of the atmosphere and the relative night-to-night changes of the FD. The measurement of the night-to-night changes is included as a standard procedure within the data taking of the FD. The main reasons for these nightly changes are dust accumulation on mirrors and filters and the slow aging of the photo multipliers tubes (PMTs) caused by the exposure to the night sky background.

In order to determine the calibration constants of the PMTs of the FD telescope after a certain time period an absolute calibration with an absolutely cal-

*e-mail: hermann-josef.mathes@kit.edu ibrated light source is done. This procedure uses a large, uniformly lit area as a light source (see fig. 1) covering the whole aperture. This so called "drum" is directly mounted to the UV filter of the FD telescope which acts also as aperture. With a diameter of over $2.40 \mathrm{~m}$ this device requires several persons to move and operate it. From this it is understandable that this absolute calibration procedure is only applied on a biennial time scale or even less frequently.

In addition and for obvious reasons it is desirable though not very practical that after any change of components either of an FD telescope or one of its relative calibration system a new absolute calibration should be performed rather close in time in order to get a new reference calibration. The resulting gap after a change in components was bridged in the past by relying on the stability of the relative calibration system and adjusting the calibration constants then in a suitable way.

\section{The Construction}

Thus we are proposing a new type of absolute calibration system which consists of a portable and time stable light source (so that it can be carried to any laboratory for detailed measurements or FD site easily) which can be utilized for a fast absolute calibration measurement. As a result of its reduced physical size, 

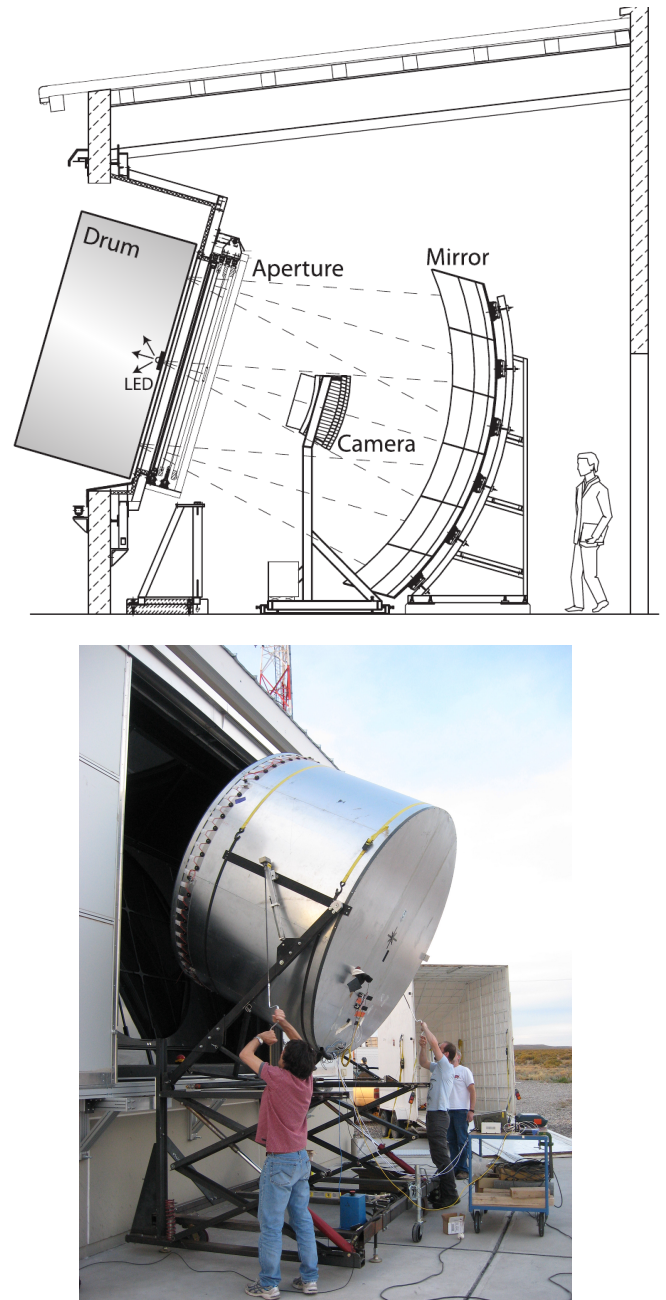

Figure 1. Scheme of the drum calibration inside the FD aperture (upper image); the drum is lifted into the aperture and several people are required for this action (lower photo).

its light output window is much smaller than the size of the aperture of the FD. Therefore the idea is to move this light source around in the aperture of the FD and by summing over all positions of the small light source the big drum should be approximated as much as possible and as necessary.

Another advantage of being small is that measurements with a closed shutter (if necessary) are possible. From earlier built light sources for the FD it has become obvious that a sufficient stabilization of the properties of the light source must be guaranteed. This includes mainly a temperature stabilization and a sufficient mechanical and long term stability of the light source.

\subsection{Mechanics and Optics}

The aperture boxes of the fluorescence telescopes are custom made with support structures constructed from Bosch Rexroth type profiles. For instance the UV filters of the FD are mounted within these profiles. The grooves of these profiles offer the possibility to mount further, lightweight equipment permanently to the aperture boxes.

Thus the new light source must be low in weight as well as the system moving this light source. We have chosen to implement the moving part by two vertically mounted profile guides (the y-axis) on which another profile guide (the $\mathrm{x}$-axis) is mounted. The $\mathrm{x}$-axis is mounted at both ends to a sliding carriage which is moved by the tooth belt of the y-axis. In the same manner, a sliding carriage at the $\mathrm{x}$-axis holds the light source. We have chosen the drylin $₫$ W profile guides from the company igus [6]. Because of their special coating and the plastic bushings of the sledges they are maintenance and lubrication free and advertised as being resistant to dirt, dust and humidity. With the chosen dimensions of the mechanical parts, the maximum weight of the light source should be below $2.5 \mathrm{~kg}$. The carriages on both axes are moved by independent motors of sufficient strength via tooth belts. During firing of light pulses a brake is used to keep everything in the selected position. This means that there is no current flowing through the motors to achieve low electromagnetic interference. Basically the carriages can be moved with sub-millimeter precision and the system applies auto-corrective measures if some steps are lost during movement.

The light source uses a $5.3^{\prime \prime}$ diameter general purpose integrating sphere of the type 4P-GPS-053-SL manufactured by labsphere [7] to create as much as possible a Lambertian distribution of the light. The sphere used has a large exit port of up to $2.5^{\prime \prime}$ diameter which can be reduced by port reducers to $2^{\prime \prime}$ and $1^{\prime \prime}$ respectively. Most of the measurements described here are performed with the $2^{\prime \prime}$ port reducer with a windowless exit port.

This sphere is oriented with the large exit port towards the optical filter of the fluorescence telescope. The other two ports hosting the LEDs and the monitoring photodiode are in a plane parallel to the filter and oriented at $90^{\circ}$ of each other (see fig. 2, right photo).

\subsection{Electronics}

One of these ports is equipped with the so called LED head, which is a temperature stabilized copper disk holding up to 3 UV LEDs (type UVLED 365-10E from Roithner LaserTechnik). The LEDs are operated with short pulses ( 1 to $8 \mu \mathrm{s}$ ) and with currents in the range 0.1 to $20 \mathrm{~mA}$. The second port also holds a photodiode (of type $S 1336$-44BQ from Hamamatsu) in a copper disk to measure the light output of the LEDs. This is mainly meant to monitor the shot-to-shot stability of the generated light pulses but not for an absolute calibration. The temperature stabilized copper disks of both heads are kept at a temperature of $30^{\circ} \mathrm{C}$ which is well above the expected temperatures during the nights when the light source is used. The heating power applied to the disk, though low, is sufficient 


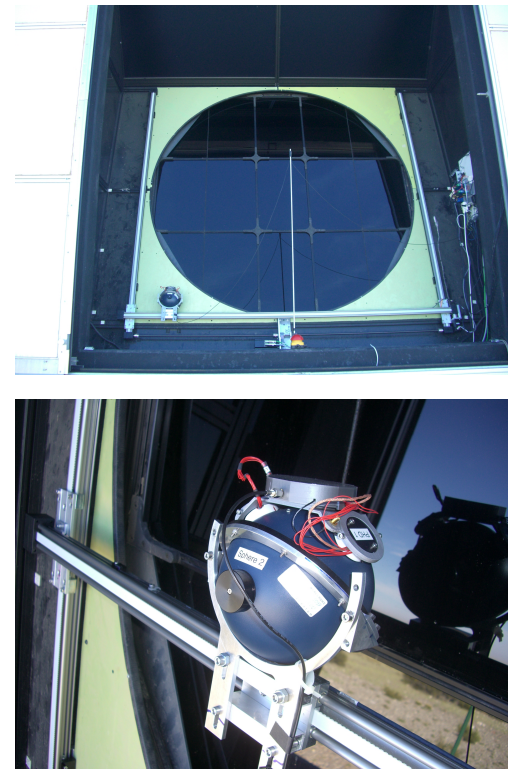

Figure 2. Full view of the XY scanner mounted to the aperture box of the Coihueco telescope 4 (upper photo). Detailed view of the integrating sphere with mounted flasher electronics (lower photo, grey case on top of the sphere), photodiode and LED head (upper right).

to heat the system to the set value of $30^{\circ} C$ starting from temperatures around $0^{\circ} C$.

The temperature control, the steering of the LEDs and the read back from the photodiode is handled by a micro controller of the MSP430 family which is placed together with all required components on a printed circuit board mounted outside the integrating sphere but close to the heads. The power supply of this board and the trigger pulse input are done via a single coaxial line. The control of the board is done using a wireless connection. The device is able to generate light pulses with a rate up to $10 \mathrm{~Hz}$ if in selftrigger mode and up to $250 \mathrm{~Hz}$ if externally triggered.

\section{Measurements}

A full measurement consists of a large number of scanning positions distributed uniformly over the area of the aperture window. For example for the case of a $2^{\prime \prime}$ wide exit window, there are roughly 2300 scan positions. First measurements were conducted with the aim to

- adjust the necessary current for the LED(s) in order to result in a certain signal height in the ADCs of the FD camera,

- determine how sparse the scan grid could be,

- and to determine the number of light flashes per scan position.

Before and after each scanning measurement, a so-called Calibration A calibration reference measurement was performed in order to track the stability of the PMT camera over the duration of the scanning measurement. This gives us also the possibility of rejecting the scanning measurement if it turns out afterwards that the drift of the PMT camera was too large. This calibration $A$ reference consists of an LED light source whose light is sent directly to the PMT camera from the center of the mirror of the telescope.

After the first measurements the LED current was set to $3 \mathrm{~mA}$ or $2.7 \mathrm{~mA}$ for an improved version of the flasher electronics which resulted in a relative magnitude of PMT amplitude of about $80-85 \%$ of the ADC scale for the brightest camera pixel.

To illustrate the second point one should keep in mind that putting circles of $5 \mathrm{~cm}$ diameter onto the aperture circle of $1300 \mathrm{~cm}$ diameter in the optimal hexagonal pattern, one would need 2301 scan points. Going to a scan distance of $6 \mathrm{~cm}$, only 1613 scan points are needed and this number reduces further to 569 if a distance of $10 \mathrm{~cm}$ is chosen.

Simulations show that the achievable accuracy with one light flash per position is sufficient. As these measurements are carried out in such a way that all PMT channels are read out after a flash, which allows for a trigger rate of roughly $1 \mathrm{~Hz}$, this results in an overall measurement time of about three quarters of an hour. This is an acceptable value which lies well within our expectations.

As the chosen exit port size of the selected integrating sphere is rather large compared to the overall size of the sphere, a certain inhomogeneity is expected. In addition, to shield the exit port from direct light from the LED port, a so called light baffle is placed inside the integrating sphere by the manufacturer. This baffle is a source of further inhomogeneities. These inhomogeneities (spatial and angular) as well as possible means to avoid them are currently under investigation in the laboratory and by simulation studies.

For the current way of mounting the integrating sphere on the XY scanning system, the orientation of the axis of maximal inhomogeneity is $45^{\circ}$ with respect to the horizontal axis. It is also expected that this should be seen during the measurements in the FD aperture. Therefore two full scans were done, one with the standard orientation of the sphere and the other one where the sphere was rotated by $180^{\circ}$ around its optical axis (defined by the vertical to the exit port plane).

\section{First Results}

So far the XY scanning calibration system was operated during two measurement campaigns, in March 2018 and in November 2018. The second campaign gave us the opportunity to repeat some of the earlier measurements with the same, untouched light source to check the stability of the overall system. It turned out that the differences between the two sets of measurements were of the order of $1 \%$, already corrected by the aging effect of the PMTs of the FD. 
Fig. 3 shows the difference of the response of the camera between a scan grid of $6 \mathrm{~cm}$ and of $10 \mathrm{~cm}$. The difference between both scan grids is less than $4 \%$. The rather regular structure comes from the arrangement of the PMTs in the camera in columns.
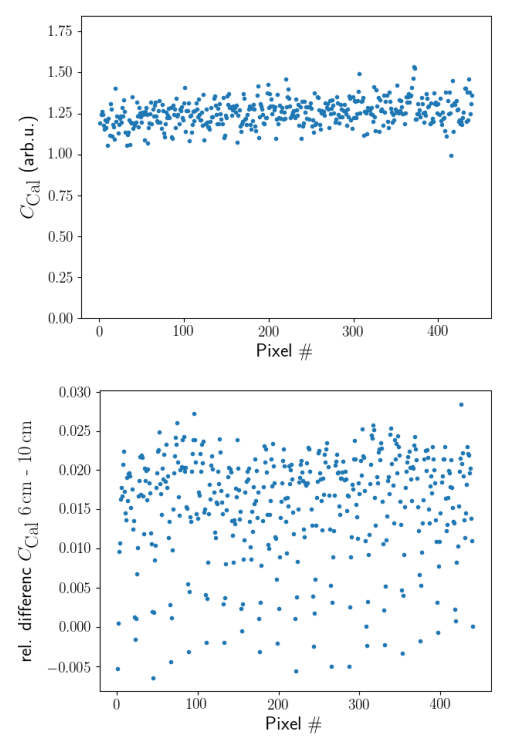

Figure 3. Calibration constants for the 440 pixels of a FD camera in arbitrary units for a sample scan grid size of $6 \mathrm{~cm}$ (upper plot) and the difference between this and $10 \mathrm{~cm}$ scan grid (lower plot).

These findings are also confirmed by simulation studies using the same two grid sizes, the expected systematic error having the same size, see fig. 4 . The shape of the distribution is however not yet compatible with the measured one.

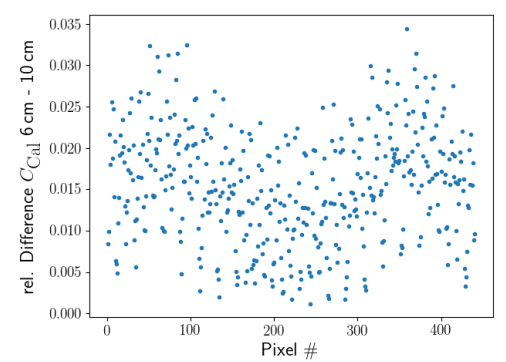

Figure 4. Difference of calibration constants of the FD camera between a scan grid of $6 \mathrm{~cm}$ and of $10 \mathrm{~cm}$ obtained from simulations

As shown in fig. 5 the effect of the baffle expressed by the difference of the two measurements is of the order of $15 \%$ when an exit port of $2^{\prime \prime}$ size is used. If the size of the exit port is reduced to for instance $1^{\prime \prime}$, the effect gets smaller and is then of the order of $9 \%$.
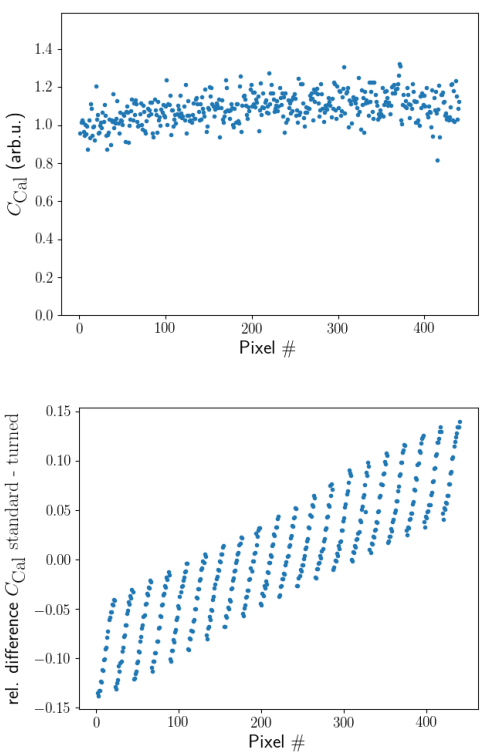

Figure 5. Calibration constants for the 440 pixels of a FD camera in arbitrary units when the sphere is mounted as seen in fig. 2, lower picture (upper plot). A slight increase from the left to the right is visible. When the sphere is rotated by $180^{\circ}$ a left-to-right decrease is expected and the difference between both configurations is shown (lower plot).

\section{Conclusion and Outlook}

Our series of measurements have shown that with an integrating sphere used as a light source and with stable operating conditions the repeatability of the measurement is rather good, i.e. the system proves already to be a valuable tool. Limiting aspects of our new technique, mainly due to some spatial inhomogeneities of the light distribution of the source can be measured and put into the simulation model to reduce the expected systematic error. There is addionionally ongoing work (both simulations and measurements) to modify the internal components of the integrating sphere (namely the baffle and the LED positioning) to reduce these inhomogeneities further.

In order to optimize the time needed for a full scan of the aperture of a single telescope, a sparser grid of scan points can be used without a significant reduction of the overall quality of the measurement. This means that it is not necessary to aim for a full coverage of the aperture.

To achieve the initial goal of providing an absolute calibration of the FD telescopes, the absolute calibration of this light source is urgently required.

So far the design of the light source used is being optimized to allow for imitating the drum measurement as much as possible. However these types of measurements are mainly targeting the degradation of the telescope due to aging or change of the transmission or reflectivity. For the analysis of the data the so called point-spread function is also important. For 
that a directional light source will be constructed and mounted to the carriage of the $\mathrm{x}$-axis in the future.

During the next campaigns further measurements will be made with the two systems already installed will be done to check the long term behavior of the system itself and to test if the system remains stable over longer periods in time. Further portable systems will be prepared for additional calibration of multiple telescopes.

Acknowledgments: We would like to acknowledge the support of this project by the BMBF grants number 05A17VK1 and 05A17PX1. This work was also supported by the Ministry of Education, Youth and Sports of the Czech Republic - Mobility Project No. 8J18DE008 and by the DAAD grant No. 57393793.

We acknowledge the contributions from $S$. Michal and
J. Šupik from the Palacký University, RCPTM, Joint Laboratory of Optics of Palacký.

\section{References}

[1] A. Aab et al., Nuclear Instrum. Methods Phys. Res. Sect. A 798, 172-213 (2015)

[2] J. Abraham et al., Nuclear Instrum. Methods Phys. Res. Sect. A 620, 227-251 (2010)

[3] H.-J. Mathes et al., Proc. of the 32nd ICRC, Beijing, China, 153-156 (2011)

[4] A. Rovero, P. Bauleo, J. Brack, J. Harton, R. Knapik, Astropart. Phys. 31 (4), 305-311 (2009)

[5] A. Aab et al., Astropart. Phys. 95, 44-56 (2017)

[6] igus ®GmbH, Spicher Str. 1a, D-51147 Köln, www.igus.de

[7] Labsphere Inc., 231 Shaker St., North Sutton, NH 03260, USA, www.labsphere.com 